\title{
Contribution of Cholinergic and GABAergic Mechanisms to Direction Tuning, Discriminability, Response Reliability, and Neuronal Rate Correlations in Macaque Middle Temporal Area
}

\author{
Alexander Thiele, ${ }^{1}$ Jose L. Herrero, ${ }^{1}$ Claudia Distler, ${ }^{2}$ and Klaus-Peter Hoffmann ${ }^{3}$ \\ ${ }^{1}$ Institute of Neuroscience, Newcastle University, Newcastle upon Tyne, NE1 7RU, United Kingdom, and ${ }^{2}$ General Zoology and Neurobiology and ${ }^{3}$ Animal \\ Physiology, Ruhr University Bochum, D-44801 Bochum, Germany
}

Previous studies have investigated the effects of acetylcholine ( $\mathrm{ACh}$ ) on neuronal tuning, coding, and attention in primary visual cortex, but its contribution to coding in extrastriate cortex is unexplored. Here we investigate the effects of ACh on tuning properties of macaque middle temporal area MT neurons and contrast them with effects of gabazine, $\mathrm{GABA}_{\mathrm{A}}$ receptor blocker. ACh increased neuronal activity, it had no effect on tuning width, but it significantly increased the direction discriminability of a neuron. Gabazine equally increased neuronal activity, but it widened tuning curves and decreased the direction discriminability of a neuron. Although gabazine significantly reduced response reliability, ACh application had little effect on response reliability. Finally, gabazine increased noise correlation of simultaneously recorded neurons, whereas ACh reduced it. Thus, both drugs increased firing rates, but only ACh application improved neuronal tuning and coding in line with effects seen in studies in which attention was selectively manipulated.

\section{Introduction}

Acetylcholine (ACh) contributes to cognitive functions, such as attention, learning, and memory, but its actions at the neuronal level in vivo remain poorly understood. Most in vivo studies have investigated effects of ACh in early sensory cortex (Sillito and Kemp, 1983; Metherate et al., 1988a,b). In primary visual cortex V1 of anesthetized cats, ACh increases neuronal orientation and direction selectivity (Sillito and Kemp, 1983; Sato et al., 1987). In V1 of anesthetized marmoset monkeys, ACh did not improve orientation tuning (Zinke et al., 2006), but it sharpened spatial tuning (Roberts et al., 2005). The latter mimics effects of attention on spatial integration properties in area V4 (Reynolds et al., 1999), V1 (Roberts et al., 2007), and middle temporal area MT (Womelsdorf et al., 2006). ACh application also increased V1 contrast sensitivity (Disney et al., 2007), again mimicking effects of attention (Williford and Maunsell, 2006; Thiele et al., 2009). Similarities between effects of attention and ACh application continue in the domain of response reliability and firing rate correlations. Attention reduces the firing rate variability of V4 and MT neurons (Mitchell et al., 2007; Niebergall et al., 2011), an

\footnotetext{
Received Feb. 6, 2012; revised Aug. 15, 2012; accepted Aug. 27, 2012.

Author contributions: A.T. designed research; A.T., J.L.H., C.D., and K.-P.H. performed research; A.T. and J.L.H. analyzed data; A.T., J.L.H., and K.-P.H. wrote the paper.

The work was supported by the Academic Research Collaboration, the German Academic Exchange Service, Medical Research Council Grant G0700976, the Biotechnology and Biological Sciences Research Council, and the ZEN program of the Hertie Foundation (to Emeritus Neuroscientist).

Correspondence should be addressed to Alexander Thiele, Institute of Neuroscience, Newcastle University, Newcastle upon Tyne, NE2 4HH. E-mail: alex.thiele@ncl.ac.uk.

DOI:10.1523/JNEUROSCI.0554-12.2012

Copyright $\odot 2012$ the authors $\quad 0270-6474 / 12 / 3216602-14 \$ 15.00 / 0$
}

effect also seen in rat V1 after basal forebrain stimulation (Goard and Dan, 2009). Attention reduces neuronal noise correlations (Cohen and Maunsell, 2009; Mitchell et al., 2009), an effect also induced by increasing cholinergic drive in rat visual cortex (Goard and Dan, 2009). Finally, ACh contributes to attentional modulation in macaque V1 (Herrero et al., 2008).

Although the effects of ACh on V1 activity are fairly well studied, the effects on tuning in extrastriate cortex are unknown. Cholinergic receptor distribution on different cells varies between V1 and V2 (Disney et al., 2006). Therefore, extrapolation of the role of ACh in $\mathrm{V} 1$ to higher cortical areas might be misleading. It is thus essential to study its role in extrastriate and higher cortical areas. Area MT is part of the extrastriate cortex in which basic tuning properties (Maunsell and Van Essen, 1983; Albright, 1984; Movshon et al., 1985; Britten et al., 1992), as well as cognitive influences, such as attention (Treue and Maunsell, 1996; Cook and Maunsell, 2002; Martínez-Trujillo and Treue, 2002), decision making (Britten et al., 1992, 1996; Thiele et al., 1999; Thiele and Hoffmann, 2008), or working memory (Bisley et al., 2004; Zaksas and Pasternak, 2006), are well studied.

We measured the effects of ACh on direction tuning, neuronal discrimination abilities, rate variability, and noise correlation. We compared the effects with those seen when $\mathrm{GABA}_{\mathrm{A}}$ receptors were blocked by gabazine, to account for effects of ACh that could be a consequence of increased firing rates, but also to revisit the role of GABAergic mechanisms in directional tuning using a more specific $\mathrm{GABA}_{\mathrm{A}}$ receptor antagonist than used previously (Thiele et al., 2004). ACh and gabazine increased firing rates, but only ACh enhanced the ability of a neuron to discriminate between different motion directions. Moreover, ACh reduced noise 
correlations, whereas gabazine increased them. Many of the effects seen with ACh application were thus comparable with the effects induced by selective attention.

\section{Materials and Methods}

All procedures were performed in accordance with the German Animal Welfare Act of July 26, 2002, the European Communities Council Directive RL 2010/63/EC, the National Institutes of Health Guidelines for the Care and Use of Animals for Experimental Procedures, and the United Kingdom Animals Scientific Procedures Act. In the present investigation, two adult anesthetized macaques (Macaca mulatta of either sex) were used.

\section{Anesthesia and analgesia}

The animals were premedicated with $0.04 \mathrm{mg} / \mathrm{kg}$ atropinsulfate. Initial anesthesia was induced by injection of ketamine hydrochloride $(10 \mathrm{mg} / \mathrm{kg}, \mathrm{i} . \mathrm{m}$.). An intravenous catheter was placed into the saphenous vein, and a bolus of $3 \mu \mathrm{g} / \mathrm{kg}$ fentanyl was given for analgesia. Animals were then continuously infused for the entire experiment with $3 \mu \mathrm{g} \cdot \mathrm{kg}^{-1} \cdot \mathrm{h}^{-1}$ fentanyl to maintain analgesia and support anesthesia. Animals were intubated [local anesthesia treatment with prilocainhydrochlorid (Xylonest spray)] through the mouth. Local anesthesia was applied to the ears [0.5\% bupivacainhydrochloride (Bupivacain)], and animals were placed in a stereotaxic apparatus. During surgery, they received additional doses of pentobarbital as needed. Animals were artificially ventilated with nitrous oxide: oxygen at a 3:1 ratio containing $0.2-0.7 \%$ halothane to maintain anesthesia. Heart rate, oxygen saturation, blood pressure, body temperature, and end-tidal $\mathrm{CO}_{2}$ were monitored and kept at physiological levels. A craniotomy was performed according to stereotaxic coordinates to allow access to cortical area MT. Area MT was then localized electrophysiologically according to its stereotaxic position and characteristic preference to stimulus movement. After all surgical procedures were completed, the animals were paralyzed with $0.1 \mathrm{mg} \cdot \mathrm{kg}^{-1} \cdot \mathrm{h}^{-1}$ vecuroniumbromide (Norcuron), and anesthesia and analgesia were maintained as described above. At the end of the experiments, the animals were killed with an overdose of pentobarbital and perfused through the heart. Details of the perfusion and histological procedures have been given previously (Distler and Hoffmann, 2001). The location of the recording sites in area MT was verified in histological sections stained for cytoarchitecture and myeloarchitecture.

\section{Visual stimulation}

A cathode ray tube $(\mathrm{CRT})$ monitor (resolution of $1600 \times 1200$ pixels at $75 \mathrm{~Hz}$ ) was placed at a distance of $53 \mathrm{~cm}$ from the animal to display visual stimuli under computer control. Initially, the receptive field (RF) of each neuron was mapped by moving a bar across a reflective sheet that was placed directly in front of the monitor. The bar was produced by a handheld lamp, and it allowed to map the RF location and borders, while its motion also served to get an initial impression of the presence or absence of direction selectivity of the recorded ensemble activity (by auditory inspection). The size of the bar could be adjusted in length and width, and its luminance was $\sim 20 \mathrm{~cd} / \mathrm{m}^{2}$ against a background of $\sim 1 \mathrm{~cd} / \mathrm{m}^{2}$. If this initial assessment yielded good ensemble spiking activity and the subjective assessment of the spike amplitudes suggested that follow on "offline" spike sorting (see below) would yield good single-unit data, we progressed as follows. After removal of the reflective sheet, direction selectivity and directional tuning in the presence and absence of drug application was determined by presenting computer-generated sinewave gratings $\left(4 \mathrm{~Hz}, 0.5 \mathrm{cycles}^{\circ}\right)$ on the CRT screen. The grating moved along 12 different directions within a circular aperture of a size that matched the ensemble RF size. For the first $500 \mathrm{~ms}$ of each trial, the screen was homogenously gray $\left(21 \mathrm{~cd} / \mathrm{m}^{2}\right)$. Thereafter, the moving stimulus was presented for $760 \mathrm{~ms}$. Stimulus contrast tested was 12.5 and $25 \%$ (Michelson contrast). We used these two contrasts to measure the effects of drug application at a contrast that is usually saturating or close to saturating (25\%) and one contrast that is likely to be nonsaturating $(12.5 \%)$ in anesthetized animals. Although $12.5 \%$ contrast can be a saturating contrast for neurons in awake macaque MT (Thiele et al., 2000), it does not seem to be the case in all awake studies (Heuer and Britten,
2002) or in anesthetized animals (Kohn and Movshon, 2003). Monitor luminance was measured with a Spectrascan PR 650 (Photo Research), and the mapping between graphics board red-green-blue output to monitor luminance was gamma corrected. Different stimulus directions and contrasts were presented randomly interleaved, while ensuring that all stimuli were presented equally often during each recording session. Stimulus presentation and data acquisition were under the control of Remote Cortex 5.95 (Laboratory of Neuropsychology, National Institute for Mental Health, Bethesda, MD) interlinked with Cheetah (Neuralynx).

\section{Recordings and drug application}

Recordings were performed with tungsten-in-glass electrodes flanked by two pipettes (Thiele et al., 2006). Neuronal activity was recorded with Neuralynx preamplifiers and amplifiers. Raw activity was bandpass filtered between 600 and $9000 \mathrm{~Hz}$. During the recording sessions, these data were thresholded to obtain multiunit activity, and the resulting spike waveforms were sampled at $30 \mathrm{kHz}$. To obtain single-unit data, offline sorting was performed based on the stored spike waveforms, using Neuralynx Spikesort3D software. All data reported here were performed based on offline-sorted spikes.

During the recording session, one pipette was filled with ACh and the other with gabazine, which were iontophoretically applied (NeuroPhore BH-2; Digitimer). Pipette opening diameter varied between 1 and $4 \mu \mathrm{m}$. Pipette resistance varied between 10 and $150 \mathrm{M} \Omega$, with most recordings at 20-80 M $\Omega$. Hold currents during control and recovery were usually $-10 \mathrm{nA}$; in rare occasions (when the pipette resistance was $10-20 \mathrm{M} \Omega$ ), it was $-40 \mathrm{nA}$. Pipette-electrode combinations were inserted into MT without the use of guide tubes. The integrity of the electrode and the pipettes were checked under the microscope before and after the recording sessions, in addition to measurements of the pipette impedance made before and after the recording at each recording site. All drugs were obtained from Sigma. The details regarding drug concentration, $\mathrm{pH}$, and application current were as follows: ACh, $0.1 \mathrm{M}, \mathrm{pH} 4.5$, application current at 5-50 nA, with most recordings at 5-30 nA; gabazine, $3 \mathrm{~mm}, \mathrm{pH}$ 3 , application current at 5-40 nA, with most recordings at 5-30 nA. Because of the range of conditions used ( 12 directions, two contrasts) and the two drugs measured, we did not obtain a dose-response curve for our neurons. Measuring dose-response curves (including additional washout periods) would have required extensive periods of time, during which cell isolation could deteriorate. Our aim was to keep the drug effects within relatively moderate limits (avoiding tripling or even doubling of firing rates if possible). We thus usually set our ejection currents to comparatively low levels, which were based on our experience from previous investigations (Thiele et al., 2004; Roberts et al., 2005; Zinke et al., 2006; Herrero et al., 2008). These currents resulted in drug-induced effects that were more limited than those reported in other studies (Sillito and Kemp, 1983; Sato et al., 1987; Roberts et al., 2005; Zinke et al., 2006; Disney et al., 2007; Katzner et al., 2011), in which ejection currents were often higher (range of $0-160 \mathrm{nA}$, most ejection currents $>40 \mathrm{nA}$ ), but they were in the range of those currents used in a recent study in V1 (Soma et al., 2012). We reported previously that "small" ACh ejection currents (5-20 nA) were necessary to enhance cognitive effects in awake primates (Herrero et al., 2008), whereas large currents can be detrimental. It is unclear how this relates to the anesthetized preparation, but we nevertheless aimed to investigate how "more moderate" drug effects alter MT response properties. Specifically, we initially set the ejection current for each recording to anywhere between 10 and $50 \mathrm{nA}$, mostly between 10 and $30 \mathrm{nA}$. If, during the initial wash-in period, the spontaneous or stimulus-driven activity appeared to increase (or decrease) too much (by visual and auditory inspection of online-monitored thresholded ensemble activity of approximately two to five neurons), we reduced the ejection current by 5-10 nA. If the activity was still increased (or decreased) too strongly (by visual and auditory inspection) after 1-2 min, we reduced the current by another 5-10 nA. If no changes occurred during this initial wash-in period, we increased the ejection current by $10-20 \mathrm{nA}$. In most cases, we did not need to change the initially chosen current. In case epileptiform activity occurred (visual and auditory inspection), we would immediately reduce the application current by at least $20-30 \mathrm{nA}$. 
We initially recorded the neuronal activity in the absence of ACh (or gabazine) for $10-15$ trials per condition ( $\sim 5$ min duration), followed by a 1-5 min "wash-in" time after the drug was turned on, followed by a recording (10-15 trials per condition) in the presence of ACh (or gabazine) ( $\sim 5$ min duration), followed by one to two recordings ( $10-15$ trials per condition), which was started after a 5 min waiting period ("washout"; because recovery usually occurs with a slight delay). Note that the criterion to increase or decrease the amount of drug applied was based on online-monitored ensemble activity, whereas the data reported here are based on offline-sorted spikes from single neurons. Because of this difference, effects of the drug that were discernible at the level of ensemble activity online did (in a few cases) not result in significant drug effects in single cells as reported in Results (see Table 1). If the activity was still stable, the above sequence was followed to record in the absence and presence of the drug (ACh or gabazine) that was not used in the first round of applications. We randomly alternated the order of the drug that was applied first. We regularly compensated for the change in current during the ejection condition by increasing the hold current of one of the two pipettes, thereby keeping the overall current identical between the "hold" and "eject" conditions.

\section{Data analyses}

Basic analysis for inclusion/exclusion of neurons. We split our data analysis into different analysis windows (for details, see below). For each of these windows, we determined whether the activity was stable over the course of the recording, i.e., whether or not "drifts" occurred. The presence of activity drifts over time was investigated by determining the firing rate associated with stimuli moving in the preferred direction (PD) (definition see below) for each trial and calculating a Pearson's correlation coefficient of rate versus trial number for these data (separately at 25 and $12.5 \%$ contrast and for the drug/no drug condition $=4$ conditions). If a significant correlation $(p<0.0125$, four conditions corrected for multiple comparisons at $p<0.05$ ) was detected, a drift was considered to be present and the data were not analyzed further (i.e., only "drift-free" cells are included in the data presented here; first step of neuronal exclusion). For those cells that were considered drift free, we tested whether drug application (drug vs no-drug), motion direction (12 directions), and stimulus contrast ( 25 vs $12.5 \%$ ) significantly affected neuronal activity (three-factor ANOVA, $p<0.05$ ). This was done separately for each of the analysis windows (see below). Neurons were excluded if they were not affected by the drug during spontaneous activity (rank-sum test, $p<$ 0.05 ) or during the stimulus-driven activity (three-factor ANOVA, $p<$ 0.05 mentioned above) (second step of neuronal exclusion). The stimulus-driven firing rate ANOVA analysis takes account of firing rates associated with any of the 12 different stimulus directions and both contrasts. Thus, it was not simply an assessment of drug effects on PD activity. Drug effects on PD activity were analyzed separately using a post hoc two-sided $t$ test $(p<0.05)$. A drug effect on stimulus-driven activity was considered to be present if the three-factor ANOVA showed a significant main effect of drug, a significant interaction between drug and contrast, a significant interaction between drug and direction, or a significant triple interaction between drug, contrast, and direction $(p<0.05)$. The ANOVA was calculated based on stimulus-driven firing rates after subtraction of spontaneous activity (spontaneous activity was calculated and subtracted separately for the drug and non-drug condition). Note that, if a neuron was tested with ACh and gabazine but, for example, only gabazine induced significant changes, then the neuron was included in the gabazine sample but not the ACh sample. If only ACh resulted in significant changes, then the opposite would apply. For neurons that were significantly affected by a drug and by the stimulus direction, we determined the PD. In our definition, the PD corresponded to the direction of motion that yielded the largest response in the absence of drug application at a stimulus contrast of $25 \%$ within the time period of interest (see below). We next determined whether the maximum firing rate of a neuron within the analysis window exceeded the spontaneous activity by $\geq 5$ spikes/s. If not, the neuron was not analyzed further (third step of neuronal exclusion). Finally, we calculated the activity for motion in the PD and the anti-preferred direction (APD; definitions are given below). From those two measures, we calculated the direction index (DI): DI =
Table 1. Number of cells included/excluded from dataset

\begin{tabular}{lllll}
\hline & ACh $0-150 \mathrm{~ms}$ & ACh $150-550 \mathrm{~ms}$ & $\begin{array}{l}\text { Gabazine } \\
0-150 \mathrm{~ms}\end{array}$ & $\begin{array}{l}\text { Gabazine } \\
150-550 \mathrm{~ms}\end{array}$ \\
\hline Included & 52 & 58 & 76 & 67 \\
Drift & 18 & 16 & 12 & 20 \\
No drug effect & 3 & 2 & 6 & 4 \\
Activity too low & 6 & 4 & 12 & 15 \\
No DI & 1 & 0 & 2 & 2 \\
Total & 80 & 80 & 108 & 108 \\
\hline
\end{tabular}

$1-$ APD/PD (baseline activity subtracted). Neurons with a DI $<0.5$ were excluded from additional analysis (fourth step of neuronal exclusion). Our exclusion steps were performed consecutively, i.e., we only analyzed drug effects, if a cell did not show drifts. We only determined whether activity levels with PD motion were $\geq 5$ spikes/s above spontaneous activity, if a drug effect was present. We only analyzed whether the DI $>0.5$ if the firing rate criterion had been positively assessed. Thus, the cell numbers listed in Table 1 are based on consecutive exclusion.

Analysis of responses in different time windows. For each neuron, we determined the latency separately for 25 and $12.5 \%$ contrast, by constructing the peristimulus time histogram (PSTH) in $1 \mathrm{~ms}$ bins, smoothed with a Gaussian kernel of $\sigma=10 \mathrm{~ms}$. We then selected the PSTH that showed the highest activity and identified the first of 15 consecutive bins, in which the activity exceeded the mean spontaneous activity more than three times the SD of the spontaneous activity. The time of the first bin fulfilling this criterion was taken to be the neuronal latency. We then calculated the neuronal activity, tuning, and $d^{\prime}$ (see below) in two different response windows. The first window ranged from 0 to $150 \mathrm{~ms}$ after response onset and captures the response transient associated with stimulus onset. The second response window ranged from 150 to $550 \mathrm{~ms}$ after response onset and corresponds to the sustained neuronal response. We used these two windows for two reasons. Our previous investigation had shown that GABA blockade affected direction selectivity mostly during the early response transient. Moreover, various studies have shown that cognitive effects on neuronal activity are more pronounced during the sustained (later) response period (Roelfsema et al., 1998; Reynolds et al., 2000; Roberts et al., 2007) (but see Ghose and Maunsell, 2002). As an additional control, we analyzed our data within fixed time windows that were set to cover the periods from 50 to 200 and 200 to $700 \mathrm{~ms}$ after stimulus onset. This control serves to determine whether a drug effect is invariant to the time after stimulus onset.

Within these defined response windows (whether related to neuronal response onset or related to stimulus onset), we determined the direction of motion that yielded the maximum activity (at $25 \%$ contrast) and defined this direction as the PD. We defined the direction of motion opposite to PD to be the APD.

\section{Analysis of directional tuning in the presence and absence of drug application}

Effect of drug application on firing rates. We calculated firing rates during spontaneous activity (i.e., while only the homogenous gray background was presented) and during two different time windows after response onset when the PD of motion was presented (0-150 and 150-550 ms after response onset, respectively). This was done separately for 12.5 and $25 \%$ contrast stimuli and for drug-applied versus no-drug-applied data. To test for differential effects between ACh and gabazine, we calculated a drug modulation index (MI) according to the following:

$$
\mathrm{MI}=\frac{\text { activity }_{\mathrm{drug}}-\text { activity }_{\text {no drug }}}{\text { activity }_{\mathrm{drug}}+\text { activity }_{\text {no drug }}}
$$

MIs reported here were based on firing rates (activity) when the preferred motion direction was presented.

Directional tuning assessment 1. To determine "when" the drug affected directionality, we calculated a time-resolved DI for each neuron at 25 and $12.5 \%$ contrast in the presence and absence of drug. We defined the PD as described above (direction of motion that yielded maximum activity at $25 \%$ contrast in the absence of drug) and calculated the neu- 
A
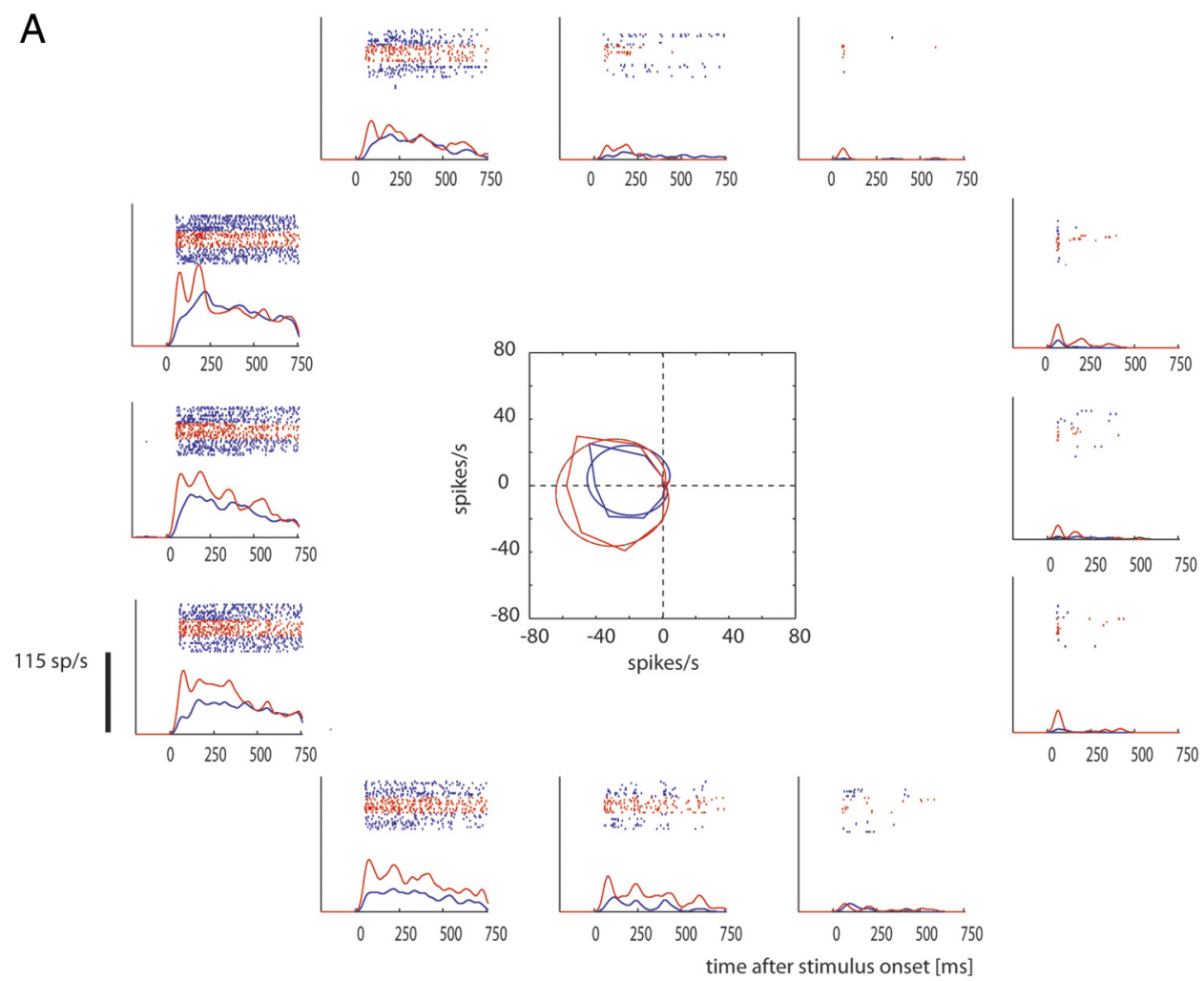

B
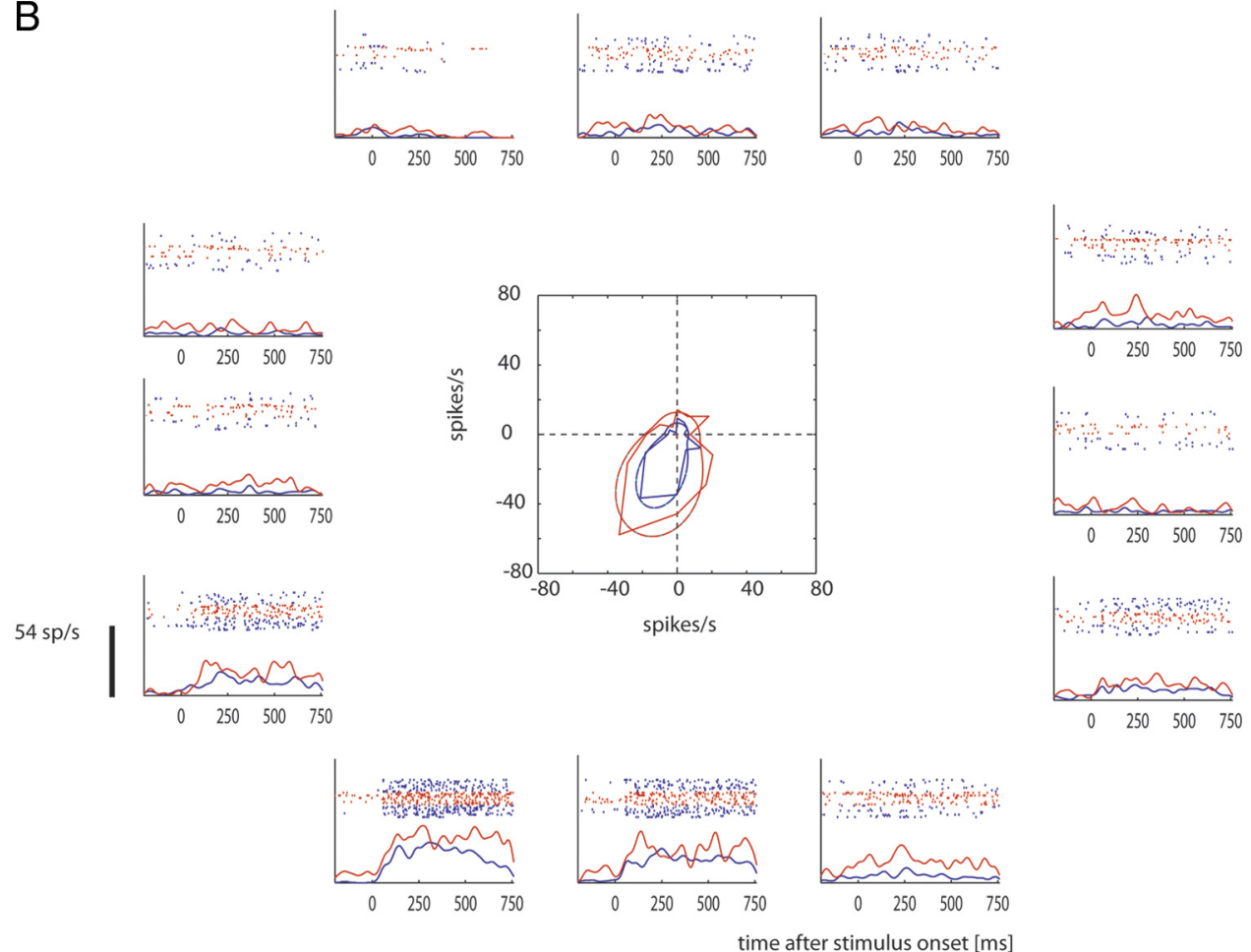

Figure 1. Effects of gabazine and ACh on direction tuning in two example neurons. $A$, Stimulus-driven activity when gabazine was applied and when it was not applied. $B$, Stimulus-driven activity, when ACh was applied and when it was not applied. Blue rasters, histograms, and tuning curves in $\boldsymbol{A}$ and $\boldsymbol{B}$ show data when no drug was applied. Red rasters, histograms, and tuning curves show data when the drug was applied. Two sets of blue rasters are shown in each histogram (flanking the red rasters). The bottom set of rasters corresponds to the initial recording, whereas the top set corresponds to the activity associated with the recovery recording. The dashed ellipsoid in the center corresponds to the wrapped Gaussian fitting, i.e., they are the tuning curves from which tuning width and amplitude were extracted.

ronal activity in $10 \mathrm{~ms}$ bins from $30 \mathrm{~ms}$ after stimulus onset until $480 \mathrm{~ms}$ after stimulus onset for the PD and the APD (baseline activity subtracted). From these activities, we calculated the DI (described above) for each time bin. This was done separately for the different drug and con- trast conditions, yielding four time-resolved DIs for each neuron. From these single-neuron DIs, the time-resolved population DI was calculated. A Wilcoxon's signed-rank test was used to determine whether (and when) the drug application had a significant effect on the DI. We used the 
false discovery rate (FDR) to account for multiple comparisons (Benjamini and Hochberg, 1995). We controlled for FDR to be at or below a value of $q=0.05$, by first ordering our $p$ values: $p(1) \leq p(2) \leq p(3) \leq \ldots \leq p(n)$, where $n$ was the number of time bins. We then accepted a threshold value of $p(r)$, where $r$ was the largest $i$ such that $p(i) \leq(i / n) \times q$. Given that we had 45 time bins per contrast, we would accept significance of an effect of the drug if at least one $p$ value was smaller than $0.05 / 45$. If so, we would alter the acceptance level and determine whether a second $p$ value was smaller than $] 2 \times(0.05 / 45)$. If so, we would alter the acceptance level again and determine whether a third $p$ value was smaller than $3 \times(0.05 / 45)$. This would be reiterated until the $n$th $p$ value was larger than $n \times$ $(0.05 / 45)$. All $p$ values until $n-1$ would be accepted as significant. In addition, we calculated the DI within response windows of $50-200$ and $200-700 \mathrm{~ms}$ after stimulus onset and determined whether these were affected by the drug application (Wilcoxon's signedrank test, $p<0.05$ ).

Directional tuning assessment 2. To determine whether drug application affected different aspects of the tuning curve, we calculated the tuning width and amplitude by fitting a wrapped Gaussian function (Swindale, 1998) to the mean response of each motion direction $\left[\chi^{2}\right.$ fitting performed under MATLAB (MathWorks)]:

$$
G(\theta)=B+A \times \sum_{n=-5}^{n=5} \exp \left(\frac{-(\theta-\rho+180 n)^{2}}{2 \times \sigma^{2}}\right)
$$

where $G(\theta)$ is the predicted response given the motion direction $(\theta), A$ is the tuning amplitude, $\sigma$ is the bandwidth, $\rho$ is the center location (the predicted preferred motion direction), and $B$ is the offset. Bandwidth was reported as full-width at half-height (FWHH). Only neurons with $>70 \%$ of variance accounted for (Carandini and Ferster, 1997) between actual and fitted values were included into the tuning widths and amplitude analysis. A Wilcoxon's signed-rank test was used to determine whether any of these tuning parameters were significantly influenced by drug application.

Drug-effect-matched samples. During our experiments, we attempted to adjust the drug ejection currents to yield changes in firing rate with $\mathrm{ACh}$ and gabazine that were on average similar. This is difficult to achieve, and we therefore did an additional control, in which we performed the above analyses based on activity-matched samples. To do so, we determined the PD activity under control conditions and under drugapplied conditions for each neuron that was recorded with ACh. We then assessed whether our gabazine sample contained neurons that had similar PD activity levels $( \pm 20 \%)$ in the control condition and that had similar drug-induced activity changes $( \pm 20 \%)$. If no such neuron was found in our gabazine sample, we excluded the neuron recorded in the ACh experiments from the ACh sample. If at least one matching "gabazine" neuron was found, we kept the ACh neuron in our ACh sample, and we added all the matching gabazine neurons to our "ACh-matched gabazine" sample. Given that any gabazine neuron could match multiple ACh neurons, we ended up with larger "ACh-matched gabazine" neuronal samples than "ACh samples." To account for this discrepancy and its possible effect on significance, we then performed sample size matching. Here we randomly drew neurons from our "ACh-matched gabazine" sample until we had equal sample sizes in both sets. We repeated this procedure 10,000 times and each time calculated $p$ values to determine whether either drug significantly affected the DI, FWHH, and tuning amplitude given the stimulus contrast and the analysis window of interest. We then took the median $p$ value that was determined for each variable and took this to reflect whether the parameter of interest was significantly affected by drug application in activity-matched neuronal samples.

Neuronal discriminability. DIs and tuning curves do not provide insight into how well a neuron can discriminate between different directions of motion, because they only measure mean responses without taking variability into account. To assess discriminability, we calculated neuronal $d^{\prime}$ values, using the response associated with PD stimuli as a reference.

We computed the neuronal $d^{\prime}$ as follows:

$$
d^{\prime}=\frac{\left(\mu_{1}-\mu_{2}\right)}{\sigma}
$$

with

$$
\sigma=\sqrt{\frac{\left(n_{1}-1\right) \sigma_{1}^{2}+\left(n_{2}-1\right) \sigma_{2}^{2}}{n_{1}+n_{2}-2}} .
$$

$\mu_{1}$ and $\mu_{2}$ represent the mean activity between activity associated with motion in PD $\left(\mu_{1}\right)$ and the activity associated with motion in any of the comparator directions $\left(\mu_{2}\right), n$ represents the number of trials, and $\sigma_{2}$ and $\sigma_{1}$ denote the SDs of the activity.

\section{Reliability of responses}

To test whether the application of ACh/gabazine showed a systematic effect on the trial-to-trial response reliability, we calculated the Fano factor $(\mathrm{FF})$, the ratio of spike count variance to mean spike count. We computed the FF for the PD motion and the APD motion when ACh/ gabazine was applied and when it was not applied. This was done in a moving time window of $50 \mathrm{~ms}$ length starting at $0 \mathrm{~ms}$ after response onset, in steps of $50 \mathrm{~ms}$ until $600 \mathrm{~ms}$ after response onset (12 time windows total). We performed a three-factor ANOVA to determine whether the stimulus (PD, APD), the drug, or the time after stimulus onset significantly affected the FF.

\section{Correlation of responses between neurons}

We calculated firing rate correlations (noise correlations) between simultaneously recorded neurons by obtaining spike counts for each trial from neurons recorded simultaneously from the same electrode from 50 to $700 \mathrm{~ms}$ after stimulus onset. We then calculated a Spearman's rank correlation coefficient between the trialwise spike counts from the two neurons for each direction of motion relative to PD of 
A
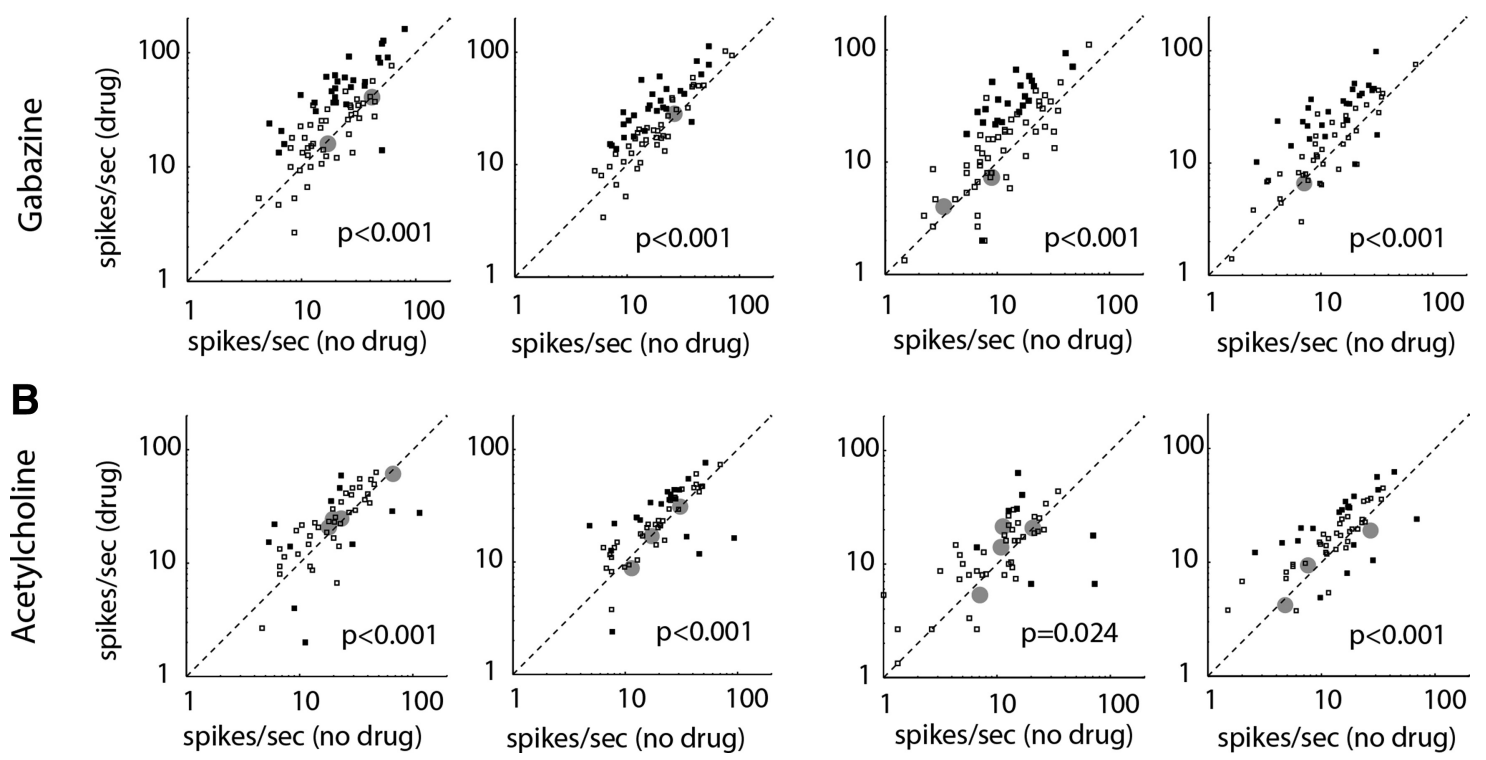

Figure 3. Effects of gabazine and ACh on activity (in spikes per second). $A$, PD activity (PD act) when gabazine was not applied (plotted along the $x$-axis) and when gabazine was applied (plotted along the $y$-axis). B, PD activity (PD act) when ACh was not applied (plotted along the $x$-axis) and when ACh was applied (plotted along the $y$-axis). $p$ values denote significance of drug effects (Wilcoxon's signed-rank test). The time window analyzed (relative to neuronal response onset) is indicated above each column. Data obtained at 25\% contrast are shown in columns 1 and 2. Data obtained at $12.5 \%$ contrast are shown in columns 3 and 4 . Square symbols indicate that stimulus-driven activity was significantly affected by the drug: black squares indicate that PD activity was also significantly affected by the drug, and gray circles indicate that stimulus-driven activity was not significantly affected (but spontaneous activity was).

A

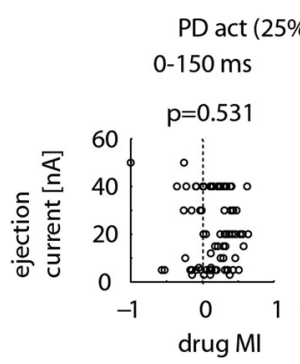

C

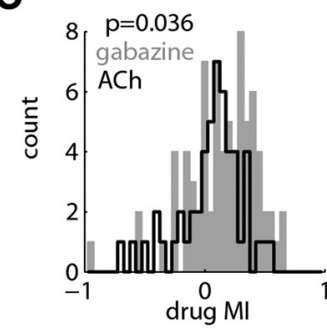

$0-150 \mathrm{~ms}(25 \%$ contrast $)$
Gabazine

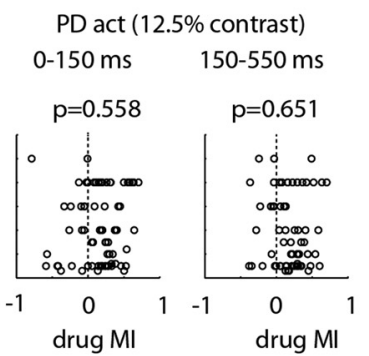

D

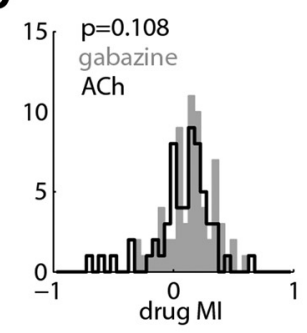

$150-550 \mathrm{~ms}$ ( $25 \%$ contrast)
E

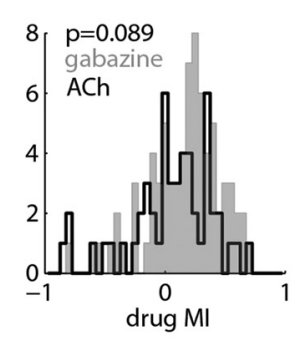

0-150 ms (12.5\% contrast)
B

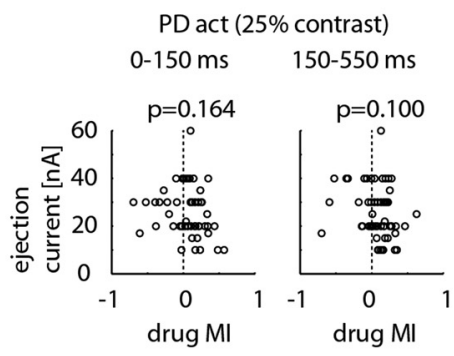

$\mathbf{F}$

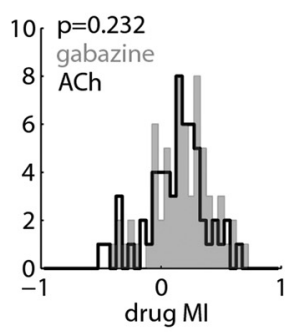

$150-550 \mathrm{~ms}$ ( $12.5 \%$ contrast)
Acetylcholine

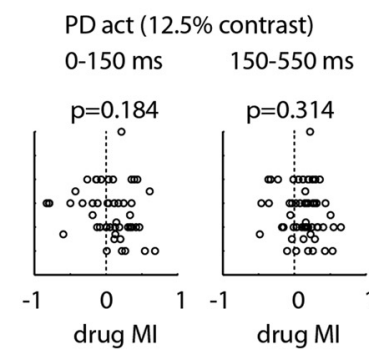

G

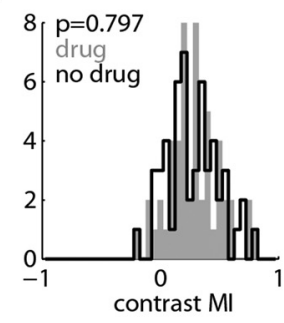

0-150 ms (ACh)

Figure 4. Effects of ejection current and drug type on stimulus-driven activity. $\boldsymbol{A}, \boldsymbol{B}$, Drug-induced change in activity (drug MI), plotted against the ejection current used for the two drugs, with different contrast and analysis windows. $p$ values show that drug MI was not significantly correlated with ejection current. $\mathbf{C}-\boldsymbol{F}$, Drug-induced activity changes (drug MI) for the two drugs, at different contrasts and time windows (gray histogram, gabazine drug Ml; black histogram, ACh drug MI). $p$ values indicate whether the two distributions significantly differed. $\mathbf{G}$, Example of contrast-induced activity changes when no drug was applied (black histogram) and contrast-induced activity changes when ACh was applied (gray histogram). Time window used was $0-150$ ms after neuronal response onset. PD act, PD activity.

neuron 1 (which was chosen arbitrarily). Given that neurons were recorded from the same electrode and because of the columnar organization of MT, neuronal pairs included here had PDs that were usually within $45^{\circ}$ of one another. The small sample size (20 pairs for ACh application, 26 pairs for gabazine application) precluded an analysis of noise correlation for neurons with more and those with less matching PD. Correlation coefficients were Fisher transformed before being subjected to a three-factor ANOVA (factor 1, direction of motion; factor 2, stimulus contrast; factor 3, drug applied/not applied). 


\section{Results}

We recorded 108 neurons during gabazine application and 80 during ACh application. As outlined in Materials and Methods, we initially determined whether or not an activity drift had occurred during the recording. For drift-free cells, we then assessed whether the drug had a significant effect on neuronal activity (during spontaneous or stimulus-driven activity). If so, we determined whether the cell was direction selective and whether stimulus-driven activity in PD exceeded spontaneous activity by $>5$ spikes/s. Because of these selection criteria (see Materials and Methods), we included data from 57 (0-150 ms response window) and 59 (150-550 ms response window) directionally selective MT neurons when ACh was applied (and when it was not applied) and from 72 (0-150 ms response window) and 71 (150$550 \mathrm{~ms}$ response window) directionally selective MT neurons when gabazine was applied and when it was not applied. Table 1 lists the cells that were excluded as a result of the exclusion criteria outlined in Materials and Methods. RF eccentricity of cells recorded ranged from $\sim 2$ to $15^{\circ}$, and RF size ranged from 3 to $18^{\circ}$ (diameter).

Figure 1 illustrates for two example cells how gabazine and ACh application could affect neuronal activity. Figure $1 A$ shows the effect of gabazine. In this example, gabazine had no significant effect on the spontaneous activity ( 0.1 spikes/s without drug vs 0.2 spikes/s with drug; $p>0.05$, signed-rank test), it slightly reduced the DI ( 0.95 without drug, 0.91 with drug), it caused a transient response even when non-PDs were presented, especially during gabazine application, it increased the tuning width $\left(113.2^{\circ}\right.$ without drug, $128.5^{\circ}$ with drug), and it increased the tuning amplitude (49.3 spikes/s without drug, 64.7 spikes/s with drug). Figure $1 B$ shows the effects of ACh application for an example cell. Here ACh significantly increased the spontaneous activity (2.4 spikes/s without drug vs 7.4 spikes/s with drug; $p<0.05$, signedrank test). It slightly increased the DI ( 0.89 without drug, 0.97 with drug), it had little effect on the tuning width $\left(62.3^{\circ}\right.$ without drug, $66.2^{\circ}$ with drug), but it increased the tuning amplitude (38.2 spikes/s without drug, 49.5 spikes/s with drug).

\section{Effects of ACh and gabazine on firing rates at the population level}

As described in Materials and Methods, cells were preselected on the basis of a significant drug effect, but the sign of the drug effect was not preselected for. Thus, in principle, increases or decreases (or no changes) could be seen at the population level. We thus first describe how the drugs affected overall neuronal activity in MT.

\section{Spontaneous activity}

Gabazine significantly increased the spontaneous activity at the population level ( $n=61, p<0.001$, signed-rank test; Fig. $2 A$ ). Fifty-six of 76 cells showed increased spontaneous activity when gabazine was applied, whereas 20 of 76 cells showed reduced spontaneous activity. Note that these changes did not have to be significant at the single-cell level, because we only required cells to show a significant drug effect on either spontaneous activity or stimulus-driven activity to be included. To outline the cells in which spontaneous activity was significantly affected by the drug, we plotted them with a different symbol (black squares) from those in which spontaneous activity was not significantly affected (gray circles). Similar effects on spontaneous activity were found when ACh was applied. ACh significantly increased the spontaneous activity ( $n=58, p<0.001$, signed-rank test; Fig. 2 ). Fortytwo of 58 cells showed increased spontaneous activity when ACh
Table 2. Drug-induced rate modulation (quantified by MIs) for different analysis time windows (relative to response onset) and stimulus contrasts

\begin{tabular}{lcrll}
\hline Drug & $\begin{array}{l}\text { Stimulus } \\
\text { contrast }\end{array}$ & \multicolumn{1}{l}{$\begin{array}{l}\text { Analysis } \\
\text { window }\end{array}$} & $\begin{array}{l}\text { Ml (median/ } \\
\text { interquartile range) }\end{array}$ & $\begin{array}{l}\text { Significance } \\
\text { (Ml group difference) }\end{array}$ \\
\hline ACh & $25 \%$ & $0-150 \mathrm{~ms}$ & $0.104 / 0.254$ & $p=0.036$ \\
Gabazine & $25 \%$ & $0-150 \mathrm{~ms}$ & $0.179 / 0.361$ & \\
ACh & $25 \%$ & $150-550 \mathrm{~ms}$ & $0.128 / 0.227$ & $p=0.108$ \\
Gabazine & $25 \%$ & $150-550 \mathrm{~ms}$ & $0.163 / 0.215$ & \\
ACh & $12.5 \%$ & $0-150 \mathrm{~ms}$ & $0.106 / 0.441$ & $p=0.089$ \\
ACh & $12.5 \%$ & $0-150 \mathrm{~ms}$ & $0.200 / 0.374$ & \\
ACh & $12.5 \%$ & $150-550 \mathrm{~ms}$ & $0.159 / 0.274$ & $p=0.232$ \\
ACh & $12.5 \%$ & $150-550 \mathrm{~ms}$ & $0.202 / 0.302$ & \\
\hline
\end{tabular}

Positive values indicate higher discharge rates during drug application. $p$ values indicate whether the respective MI distributions were significantly different (rank-sum test).

\begin{tabular}{|c|c|c|c|}
\hline Drug condition & $\begin{array}{l}\text { Analysis } \\
\text { window }\end{array}$ & $\begin{array}{l}\text { Contrast MI (median/ } \\
\text { interquartile range) }\end{array}$ & $\begin{array}{l}\text { Significance } \\
\text { (Ml group difference) }\end{array}$ \\
\hline ACh not applied & $0-150 \mathrm{~ms}$ & $0.249 / 0.265$ & $p=0.797$ \\
\hline ACh applied & $0-150 \mathrm{~ms}$ & $0.276 / 0.269$ & \\
\hline ACh not applied & $150-550 \mathrm{~ms}$ & $0.183 / 0.218$ & $p=0.635$ \\
\hline ACh applied & $150-550 \mathrm{~ms}$ & $0.145 / 0.312$ & \\
\hline Gabazine not applied & $0-150 \mathrm{~ms}$ & $0.223 / 0.308$ & $p=0.644$ \\
\hline Gabazine applied & $0-150 \mathrm{~ms}$ & $0.217 / 0.377$ & \\
\hline Gabazine not applied & $150-550 \mathrm{~ms}$ & $0.187 / 0.235$ & $p=0.284$ \\
\hline Gabazine applied & $150-550 \mathrm{~ms}$ & $0.145 / 0.309$ & \\
\hline
\end{tabular}

Positive values indicate higher discharge rates when high-contrast stimuli (25\%) were presented. $p$ values indicate whether the respective MI distributions were significantly different (rank-sum test).

was applied, whereas 16 of 58 cells showed reduced spontaneous activity (Fig. $2 B$ ). As in Figure $2 A$, spontaneous activity significantly affected by the drug is plotted as a black square, whereas spontaneous activity not significantly affected by the drug is plotted as a gray circle. Some data points are close to the unity line, but spontaneous activity was nevertheless significantly affected by the drug. The large number of trials available to assess spontaneous activity yielded significance, despite overall small differences $(>2 \times 12 \times 10$ trials per drug condition). Overall spontaneous activity in the absence of drug application was lower (mean of 2.8 spikes/s) than that reported in other studies (Maunsell and Van Essen, 1983; Britten et al., 1993).

\section{Stimulus-driven activity}

We analyzed stimulus-driven responses in PD in two separate response windows, one corresponding to the transient response (0-150 ms after response onset) and the other to the sustained response (150-550 ms after response onset). The exact number of cells that showed increased activity during drug application for the PD of motion varied marginally between response windows and different stimulus contrast, so we do not report them individually here. They can be deduced from Figure 3 . Note that significant drug effects were assessed by means of a two-factor ANOVA (see Materials and Methods). This means that the drug could be effective across all stimulus motion directions, or only at a few stimulus directions, which may or may not include the PD. We therefore assessed the overall $p$ value of the drug effect obtained from the ANOVA, which yields the cells that showed any drug effect during stimulus presentation, and also performed a post hoc analysis to determine whether the drug was effective when the PD was presented. If the former was the case but the latter not, we use a different symbol in Figure 3, as detailed below. The plots in Figure 3, $A$ and $B$, separately show the number of cells 
A

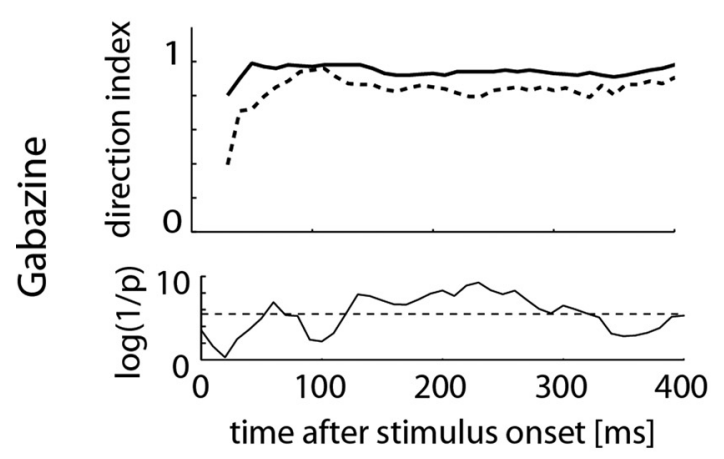

B

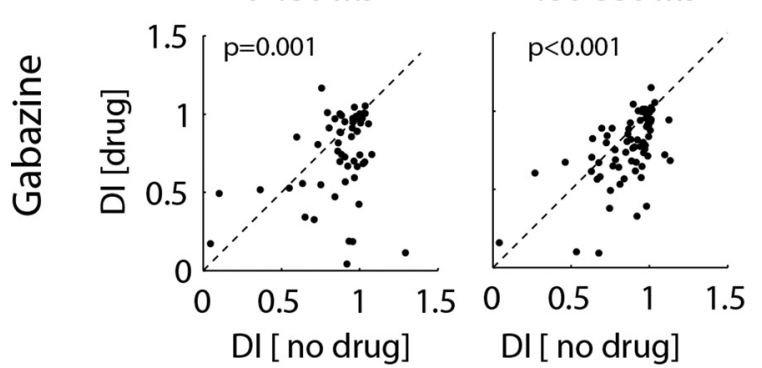

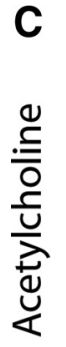

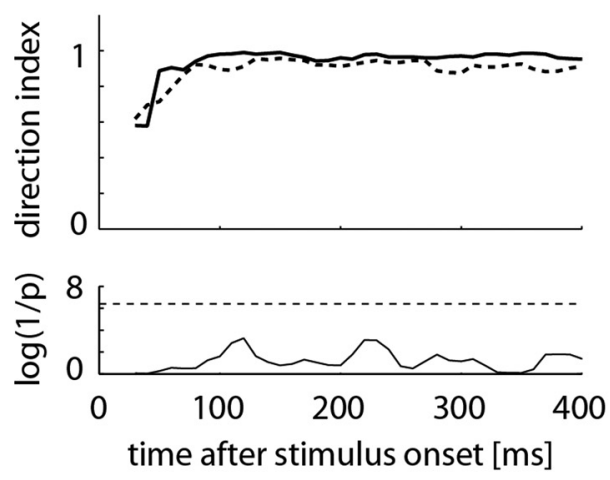

D

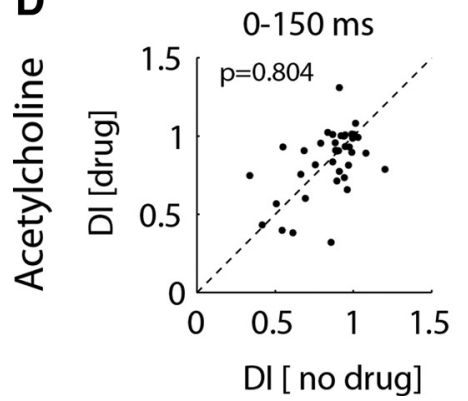

$25 \%$ contrast

150-550 ms

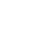


0-150 ms window

$25 \%$ contrast
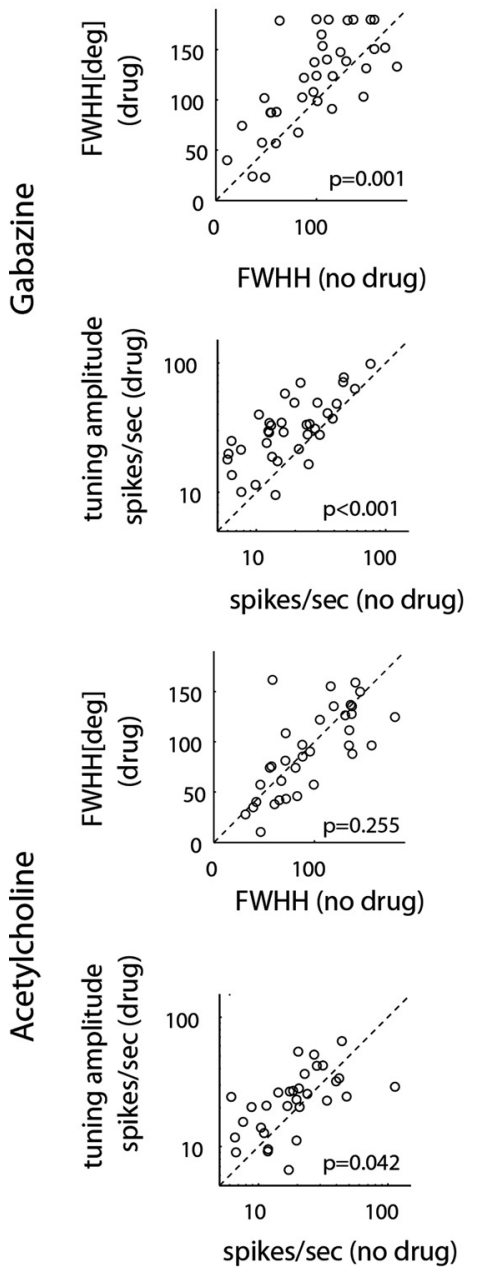

$12.5 \%$ contrast

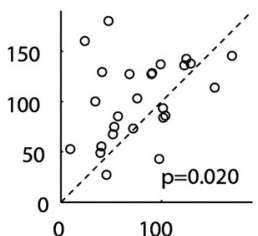

FWHH (no drug)
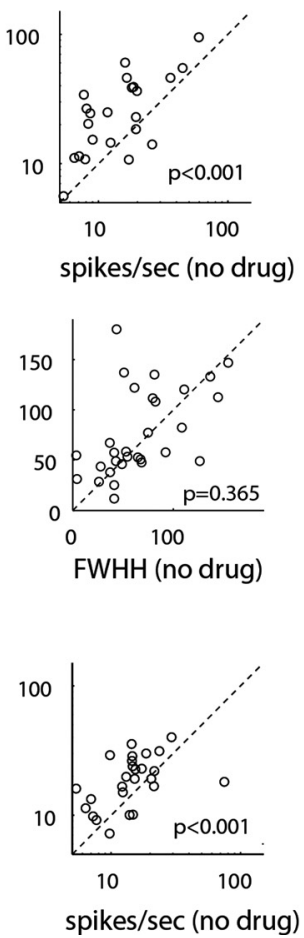

150-550 ms window

$25 \%$ contrast

$12.5 \%$ contrast
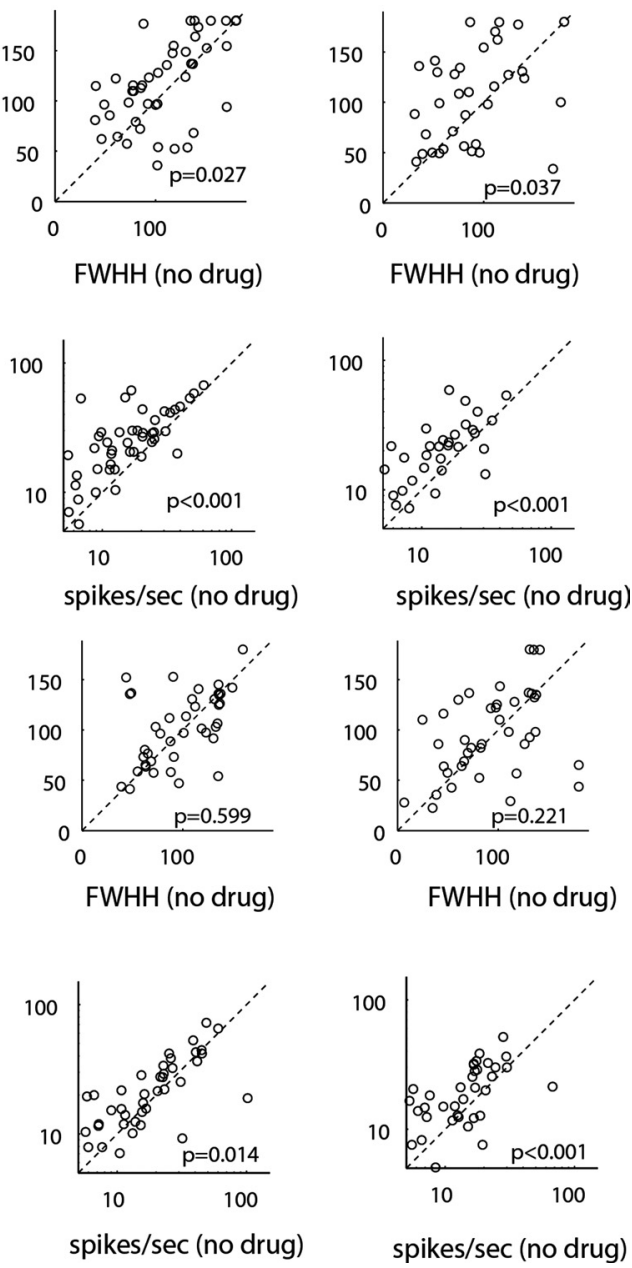
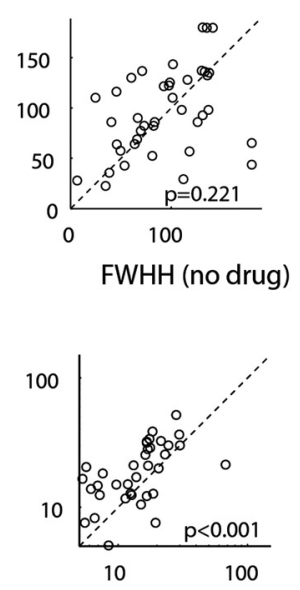

spikes/sec (no drug)

Figure 6. Influence of gabazine and ACh on tuning width and tuning amplitude. The top two rows show data relating to gabazine application (row 1, tuning width; row 2, tuning amplitude), and the bottom two rows show data relating to ACh application (row 3, tuning width; row 4, tuning amplitude). Columns 1 ( $25 \%$ contrast stimuli) and 2 ( $12.5 \%$ contrast stimuli) show data relating to the early response window, and columns 3 (25\% contrast stimuli) and 4 ( $12.5 \%$ contrast stimuli) show data relating to the late response window. Data obtained in the no-drug condition are plotted along the $x$-axis in each subplot, and those obtained with drug applied are plotted along the $y$-axis in each subplot. $p$ values indicate level of significance for the drug/no-drug comparison (signed-rank test).

levels tested $(p<0.05$, signed-rank test; Fig. $3 A, B)$. As described in Materials and Methods, we did not establish a dose-response curve for our neurons and the ejection currents did vary between recordings (see Materials and Methods). The strength of effects could thus be correlated with the ejection currents used, even if we aimed for moderate drug effects across the sample of neurons. Contrary to this suggestion, Figure $4, A$ and $B$, demonstrates that there was no correlation between ejection current and drug efficacy in our dataset ( $p \geq 0.1$, Pearson's correlation coefficient).

To quantify the differential effect between ACh and gabazine on stimulus-driven activity, we calculated MIs for the different time windows, contrasts, and drugs conditions. The means and SDs of these MIs are listed in Table 2. Gabazine application induced significantly larger response changes in the early response window at high contrast than ACh did $(p=0.036$, rank-sum test). This is illustrated by Figure $4 C$, in which drug-induced response MIs for $25 \%$ contrast stimuli during the early response window are shown. No differences in drug efficacy were found at
$25 \%$ contrast for the late response period or for the $12.5 \%$ contrast in either response window (Fig. 4C-F).

We used two different contrasts (12.5 and 25\%) that were fixed throughout our study. Although these contrasts may be saturating for some neurons, we found that they yielded significantly different firing rates in the absence of drug application for the populations of cells $(p<0.001$, all samples and response windows, signed-rank test). Specifically, for our cell sample recorded with and without ACh, the $25 \%$ contrast stimulus resulted in a $66 \%$ (median, no ACh) and 76\% (median, ACh applied) increase of firing rates compared with the $12.5 \%$ contrast stimulus (preferred motion direction; interquartile range, 107 and 105\%, respectively) for the early response window. It resulted in a $44 \%$ (median, no ACh) and 29\% (median, ACh applied) increase of firing rates compared with the $12.5 \%$ contrast stimulus (interquartile range, 63 and $62 \%$, respectively) for the late response window. The respective numbers for our gabazine cell sample were a 57\% increase (no gabazine) and 53\% 
Gabazine 0-150 ms window

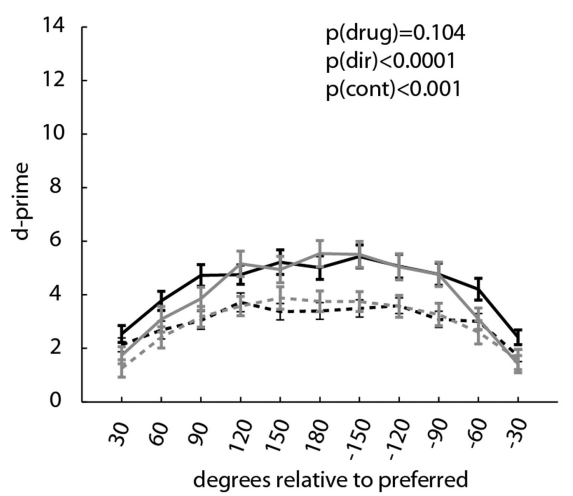

ACh 0-150 ms window

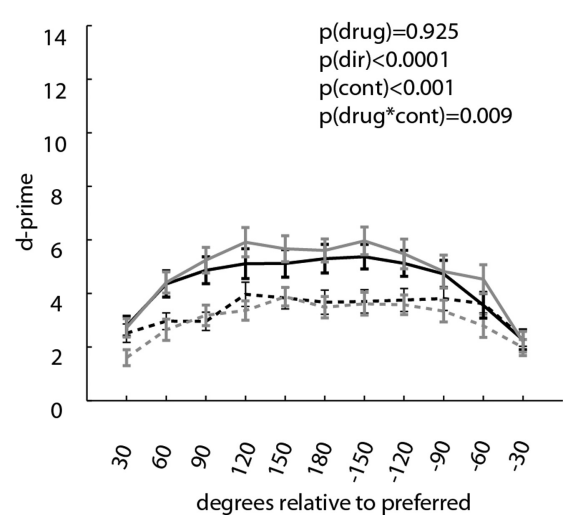

Gabazine 150-550 ms window

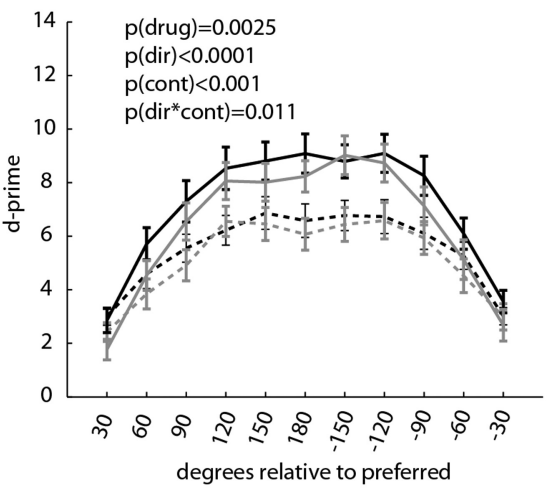

ACh $150-550$ ms window

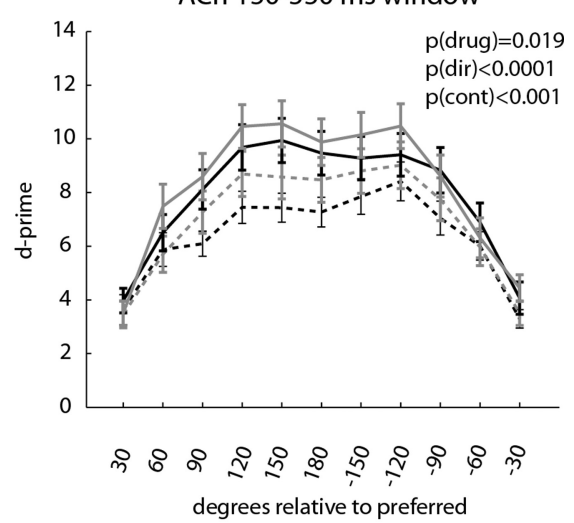

cation significantly increased the FWHH and amplitude for both response windows at both stimulus contrasts (signed-rank test, for exact $p$ values, see Fig. 6). Conversely, ACh application had no significant effect on the FWHH in any response window or at any contrast. However, ACh significantly increased the tuning amplitude for both response windows at both stimulus contrasts (signed-rank test, for exact $p$ values, see Fig. 6).

Note that the number of entries in Figure 6 does vary and is lower than the reported number of cells included in the overall analysis. This is because the quality of fits could vary for the different response windows and contrasts. To obtain reasonable tuning estimates, the quality of the fit had to be acceptable, whereby the variance accounted for by any drug-related and no-drug-related fit had to be $>70 \%$ (see Materials and Methods).

\section{Activity-matched $\mathrm{ACh}$ and gabazine sample analysis}

All the above analyses of effects of gabazine and ACh were based on our entire sample of cells that passed our inclusion criteria (see Materials and Methods). Although drug effects between gabazine and ACh on PD activity were only significantly different for the early response window at $25 \%$ contrast, the distributions shown in Figure $4 C-F$ and the values presented in Table 2 suggest that gabazine resulted in somewhat stronger increases in firing rates at the population level than ACh ap-

increase (gabazine applied) (interquartile range, 116 and 132\%, respectively) for the early response window and a $46 \%$ increase (no gabazine) and 33\% increase (gabazine applied) (interquartile range, 74 and $87 \%$, respectively) for the late response window. We calculated MI distributions for all these contrast comparisons and determined whether the contrast MI distributions differed between drug applied and drug not applied (Table 3 ). All the distributions were significantly offset from 0 ( $p<0.001$, ranksum test), but they did not differ significantly from one another (the smallest $p$ value was $=0.285$, Wilcoxon's signed-rank test), which demonstrates that the drugs were equally effective at 12.5 and $25 \%$ contrast. This pattern was found for both response windows and both drugs. An example of the two contrast MI distributions for ACh not applied and ACh applied (early response window) is shown in Figure $4 G$.

\section{Directional tuning}

Gabazine application significantly decreased the DI at 12.5 and $25 \%$ contrast (Fig. $5 A, B$ ). This effect was significant for the early response window and the late response window for both contrast levels (Fig. 5B). ACh did not significantly affect the DI at any point in time at 12.5 or $25 \%$ contrast (Fig. $5 C, D$ ).

To further quantify the effects of drug application on directional tuning, we fitted a wrapped Gaussian to the mean firing rates (see Materials and Methods), which yielded data relating to tuning widths (FWHH) and tuning amplitude. Gabazine appli- plication did. We therefore performed the above analyses on activity-matched samples as described in Materials and Methods. Using these matched samples, we found that ACh and gabazine significantly increased the tuning amplitude at both contrasts and in both analysis windows ( $p<0.01$, signed-rank test). Only gabazine significantly increased the FWHH (both analyses windows and both contrasts, all $p<0.05$, signed-rank test), whereas ACh did not have an effect in either analysis windows at either contrast (all $p>0.1$, signed-rank test). Gabazine significantly reduced the DI at 12.5 and $25 \%$ contrast in both analysis windows $(p<0.01$, signed-rank test). For the activity-matched sample, ACh did not affect the DI at 25 or $12.5 \%$ contrast in the early or late analysis window. Thus, all analyses performed with the activity-matched samples yield results that are qualitatively and quantitatively similar to those performed on our entire cell sample. The differential effects on tuning functions seen between gabazine and ACh application therefore cannot be accounted for by larger activity increases induced by gabazine application.

\section{Stimulus discriminability}

The above analyses gave insight into directional tuning measures based on mean responses, but these measures do not provide insight into neuronal discriminability. To assess the latter, we calculated neuronal $d^{\prime}$, which takes differences in mean activity and the neuronal variability into account. We used the response associated with PD stimuli as our reference and calculated $d^{\prime}$ for 
all the other directions of motion. This was done separately for the two response windows (relative to the response onset of a neuron, i.e., latency; see Materials and Methods), for each contrast and for the drug and no-drug conditions. The outcome of this analysis is shown in Figure 7. During the early response period, $d^{\prime}$ values were overall lower compared with the late response period. This is most likely attributable to a response transient that occurs in some neurons with stimulus presentation regardless of the direction of motion. The stimulus transient has motion energy in opposing stimulus directions across a range of spatial frequencies, and thus reduced $d^{\prime}$ values for the early response period are not surprising. However, stimulus direction and contrast still had a significant effect on neuronal $d^{\prime}$. Gabazine did not affect neuronal discriminability during the early response $[p>0.05$, three-factor repeated-measures (RM) ANOVA]. ACh increased neuronal discriminabilty in the early response window for high-contrast but not for low-contrast stimuli (drug $\times$ contrast interaction: $p=$ 0.009, three-factor RM-ANOVA). During the late period, gabazine application significantly reduced $d^{\prime}$ values ( $p=0.0025$, threefactor RM-ANOVA), i.e., gabazine reduced the ability of a neuron to discriminate between preferred and other directions of motion. Conversely, ACh significantly increased the neuronal $d^{\prime}$ during the late response period ( $p=0.019$, three-factor RM-ANOVA), i.e., ACh increased the ability of a neuron to discriminate between preferred and other directions of motion.

\section{Reliability of neuronal responses (FFs)}

Figure 8 shows the time-resolved FFs (relative to the response onset of the neuron) for PD and APD motion for both drug and contrast conditions. The solid straight horizontal lines show the FF during spontaneous activity ( -50 to $0 \mathrm{~ms}$ before stimulus onset) during the no-drug and drug conditions. FFs were reduced after response onset, in line with recent reports (Churchland et al., 2010); they remained at somewhat higher levels than those reported from awake monkeys but are similar to those reported from area MT in anesthetized monkeys (Churchland et al., 2010). Gabazine significantly increased FFs at 12.5 and $25 \%$ contrast $(p<0.001$, three-factor ANOVA, drug main effect). There was no effect of stimulus direction or time after response onset on FFs at either contrast ( $p>0.05$, three-factor ANOVA). ACh did not affect FFs at 25 or $12.5 \%$ contrast (main effect of drug $>0.05$, three-factor ANOVA), none of the other factors had a significant main effect on FFs, and there were no interactions between any of the effects. Our selection criterion required neurons to be significantly affected by the drug application. This selection was based on firing rates. It could be the case that the drug does not affect the firing rate of a neuron but still alters the FF. To account for this possibility, we included the (few) neurons that were not affected by the drug in the analysis. The effect of the drugs on FFs did not change when these neurons were included. Gabazine still resulted in a significant increase in FF at both contrasts $(p<0.01$,
$25 \%$ contrast
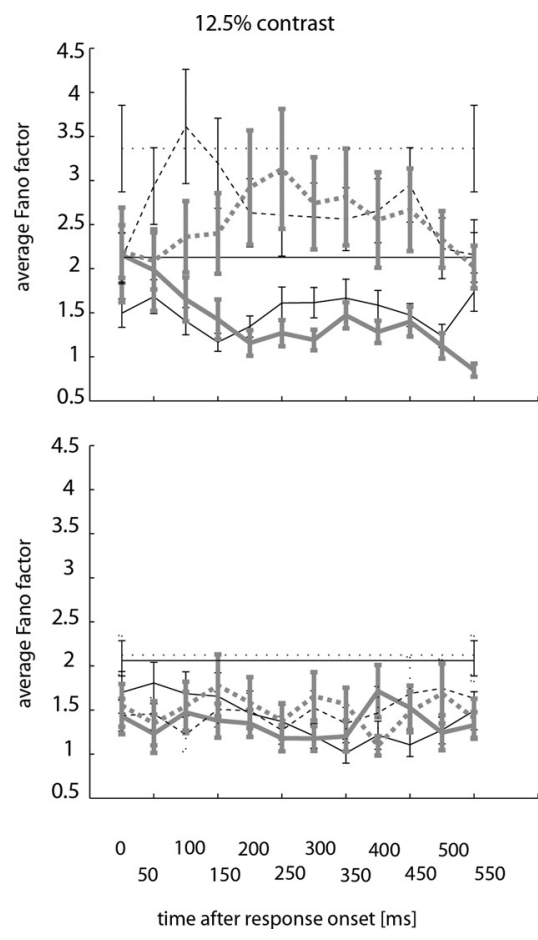

Figure 8. Influence of gabazine and ACh on response reliability. Response reliability (FF) was measured in windows of $50 \mathrm{~ms}$ length, shifted in steps of $50 \mathrm{~ms}$ after response onset ( $x$-axis). The top row shows the results when gabazine was not applied and in was applied for stimuli moving in PD (black lines) and APD (gray lines). The bottom row shows the equivalent results for lines show data for the no drug condition, and dashed lines show data for the drug condition. Error bars show SEM. The black straight horizontal lines show spontaneous FF and SEM (solid line, drug not applied; dotted line, drug applied).

three-factor ANOVA, drug main effect). ACh still did not alter the FF when neurons not significantly affected by drug application were included ( $p>0.05$, three-factor ANOVA).

Noise correlation between simultaneously recorded neurons

We measured noise correlation between simultaneously recorded neurons as a function of motion direction relative to PD and as a function of drug application. We included pairs of neurons into the analysis if the PD of the two neurons did not differ by $>45^{\circ}$, i.e., both neurons preferred more or less the same direction of motion. We did not find a significant main effect of contrast for any of our datasets $(p>0.2$, three-factor ANOVA, main effect of contrast) and thus pooled across different contrasts. Figure 9 shows the results for the two different drugs applied. Gabazine significantly increased noise correlations (significant main effect of drug, $p<0.001$, three-factor ANOVA). We did not find a main effect of direction $(p=0.74$, three-factor ANOVA) or an interaction between direction and $\operatorname{drug}(p=0.15$, three-factor ANOVA) in our dataset. Conversely, ACh significantly reduced noise correlations (significant main effect of drug, $p<0.001$, three-factor ANOVA). Stimulus direction had no effect on noise correlations $(p=0.85$, three-factor ANOVA), and there was no significant interaction between direction and drug ( $p=0.91$, three-factor ANOVA). In line with the drug efficacy argument made in the FF section, we recalculated noise correlations with neurons included that did not show significant drug-induced alterations of firing rates. The additional analysis did not change the outcome, i.e., ACh still significantly reduced noise correlations $(p<0.001)$, whereas Gabazine increased it $(p<0.001)$. Thus, gabazine increased noise correla- 


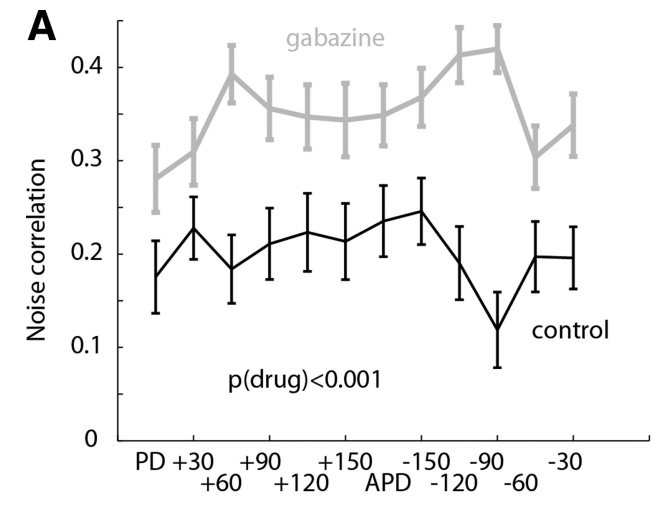

stimulus direction [deg] relative to preferred

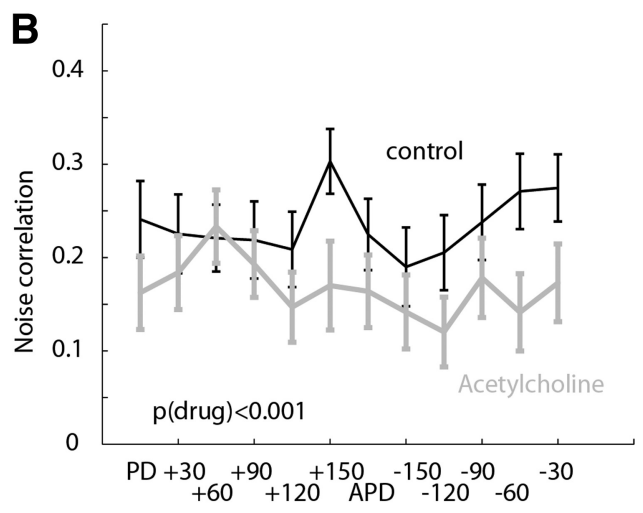

stimulus direction [deg] relative to preferred

Figure 9. Influence of gabazine $(\boldsymbol{A})$ and $\mathrm{ACh}(\boldsymbol{B})$ on noise correlations for stimuli moving in different directions relative to PD. Gray lines and error bars show the noise correlations when the drug was applied, and black lines and error bars show the noise correlations when no drug was applied. Noise correlation data for the different contrasts are pooled, because there was no difference between 25 and $12.5 \%$ contrast stimuli. $p$ values denote whether drug application had a significant effect on noise correlations. Error bars show SEM.

tion at all contrasts and for all directions, whereas ACh reduced noise correlations at all contrasts and for all directions.

\section{Discussion}

Here we report the effects of ACh and gabazine application on MT neuron firing rates, tuning properties, response reliability, and rate correlations. Gabazine and ACh both increased stimulus-driven firing rates but had very different effects on neuronal tuning, discriminability, response reliability, and neuronal correlations. Gabazine deteriorated tuning, as assessed by DI, tuning width, and discriminability. Conversely, ACh had no negative effects on DI, tuning width, and improved discriminability. Gabazine resulted in reduced response reliability for PD and APD motion direction stimuli. ACh did not result in reduced response reliability when PD motion stimuli were presented. Gabazine application increased noise correlations, whereas ACh application decreased noise correlations. Thus, relatively similar increases in firing rates by gabazine and ACh can translate into very different effects on other response characteristics.

\section{Interaction with anesthesia}

Here we recorded the effects of ACh and gabazine application on tuning functions in MT under anesthesia. We decided to perform these experiments in the anesthetized animal, because this preparation allows investigating more stimulus conditions with and without drug applied than is feasible in the awake animal, especially in animals performing attention-demanding tasks. How- ever, anesthesia interacts with the cholinergic and GABAergic systems (Anthony et al., 1989; Yamakura et al., 2001; Tassonyi et al., 2002), which may have implications for our results, especially regarding our findings in relation to noise correlations and FFs. If halothane induced a synchronized brain state (Harris and Thiele, 2011), then noise correlations might be increased overall, and alterations seen with, for example, ACh might be much larger than what would occur in awake animals. However, the noise correlations in the absence of drugs were relatively close to those reported in awake animals (Zohary et al., 1994; Thiele and Hoffmann, 2008). It will still be important to perform similar drugrelated experiments in the awake preparation, but, based on our experience in V1, these have to be restricted to fewer stimulus conditions, whereby detailed tuning properties are much harder to investigate than under anesthesia.

\section{Directional tuning}

GABAergic mechanisms contribute to directional tuning in V1 (Sillito, 1977; Sato et al., 1995; Crook et al., 1998; Murthy and Humphrey, 1999; Roerig and Kao, 1999). It was argued that neurons in macaque area MT inherit their direction selectivity from neurons in areas V1, V2, and V3 (Movshon and Newsome, 1996), because cells that project to MT are already directionally selective. Blocking $\mathrm{GABA}_{\mathrm{A}}$ receptors in area MT with bicucullinemethiodide (BMI) nevertheless demonstrated that MT cells often generate directional selectivity de novo (Thiele et al., 2004). BMI reduced direction selectivity during the early response period, whereas it was preserved in later response periods. In line with these findings, we show here that gabazine reduced directional tuning of MT neurons. Thiele et al. (2004) also reported that BMI application increased the tuning width of MT neurons, which we replicate using gabazine. Despite these similarities, some of the gabazine effects differed from those reported previously (Thiele et al., 2004). First, effects on DI were smaller. Second, we did not see the main changes on DI to occur during the initial response period but also during the later response period. Although the differences are mostly quantitative, they are still noteworthy. Thiele at al. (2004) used BMI, which also blocks small-conductance calcium-activated potassium channels (Khawaled et al., 1999), thereby affecting the slow afterhyperpolarization. Gabazine is highly specific for $\mathrm{GABA}_{\mathrm{A}}$ receptors, and the differences found between this study and the study by Thiele et al. (2004) could thus be attributable to differentially affecting SK channels.

Based on previous studies in V1 (Sillito and Kemp, 1983; Sato et al., 1987; Murphy and Sillito, 1991), we predicted that ACh application would result in sharpened directional tuning in MT neurons. However, we found no effect of ACh application on either the DI or tuning width. We found, however, a significantly increased tuning amplitude. The latter was also found with gabazine application. Thus, increased stimulus-driven responses, which occurred with gabazine and ACh application, resulted in reduced directional tuning when $\mathrm{GABA}_{\mathrm{A}}$ transmission was blocked but in unchanged directional tuning when cholinergic receptors were activated. Because ACh did not affect the response variability when motion in PD was presented, the increased tuning amplitude translated into increased neuronal discriminability. The latter was not found when gabazine was applied, most likely because gabazine strongly increased response variability, which deteriorates the signal.

ACh contributes to attentional mechanisms (Gill et al., 2000; Parikh et al., 2007; Herrero et al., 2008; Parikh and Sarter, 2008; Deco and Thiele, 2009, 2011), and the effects seen on directional tuning with ACh application are similar to those reported when 
attention is directed to the RF of the neuron under study, namely multiplicative changes in response amplitude without affecting tuning width (McAdams and Maunsell, 1999; Martinez-Trujillo and Treue, 2004; Busse et al., 2008; Womelsdorf et al., 2008). These results are not simply the consequence of increased overall activity, because $\mathrm{GABA}_{\mathrm{A}}$ receptor blockade had very different effects (e.g., increased tuning width). This suggests that attentional modulation affects excitatory and inhibitory circuits equally, ensuring that excitatory drive is balanced by appropriate inhibition, as suggested by recent models of attention (Ghose, 2009; Lee and Maunsell, 2009; Reynolds and Heeger, 2009).

\section{Response reliability}

Application of gabazine resulted in reduced response reliability, whereas application of ACh had little effect on response reliability. Previous studies in V4 (Mitchell et al., 2007, 2009) have observed increased response reliability with attention. Although this was also seen after basal forebrain stimulation in anesthetized rats, which increases ACh drive cortically and subcortically (Goard and Dan, 2009), the effects in V1 were attributable to mechanisms along the subcortical processing pathway (Goard and Dan, 2009). Thus, it appears that ACh does not contribute directly at the cortical level to attention-induced improvements of response reliability. Increased response reliability with attention might be attributable to glutamatergic (feedback) drive, which could stabilize the local network and make it more resistant to slow response fluctuations induced by unspecific inputs.

\section{Noise correlations}

Despite having similar effects on firing rates, gabazine and $\mathrm{ACh}$ application had opposite effects on noise correlations in MT. Gabazine increased noise correlations, whereas ACh reduced noise correlations. Reduced noise correlation was also reported in V1 after basal forebrain stimulation in anesthetized rats, and these effects were mediated by muscarinic receptors within V1 (Goard and Dan, 2009). Attention reduces noise correlations in V4 (Cohen and Maunsell, 2009; Mitchell et al., 2009), and the results reported here and those reported by Goard and Dan (2009) suggest that ACh might be an important contributor to this attention-induced noise correlation reduction. To assert this, it will be necessary to record from multiple neurons while animals perform attention-demanding tasks and determine noise correlations with and without cholinergic blockade.

\section{Conclusions}

In summary, gabazine and ACh application both resulted in increased firing rates but had very different effects on directional tuning functions, discriminability, response reliability, and noise correlations. Some, but not all, of the effects seen with ACh application were reminiscent of those reported in animals performing attention-demanding tasks. This further supports the notion that ACh makes important contributions to attentional modulation, but it also highlights that our understanding of the mechanisms of attention is still very incomplete.

\section{References}

Albright TD (1984) Direction and orientation selectivity of neurons in visual area MT of the macaque. J Neurophysiol 52:1106-1130.

Anthony BL, Dennison RL, Aronstam RS (1989) Disruption of muscarinic receptor-G protein coupling is a general property of liquid volatile anesthetics. Neurosci Lett 99:191-196.

Benjamini Y, Hochberg Y (1995) Controlling the false discovery rate: a practical and powerful approach to multiple testing. J R Stat Soc Ser B Stat Methodol 57:289-300.
Bisley JW, Zaksas D, Droll JA, Pasternak T (2004) Activity of neurons in cortical area MT during a memory for motion task. J Neurophysiol 91:286-300.

Britten KH, Shadlen MN, Newsome WT, Movshon JA (1992) The analysis of visual motion: a comparison of neuronal and psychophysical performance. J Neurosci 12:4745-4765.

Britten KH, Shadlen MN, Newsome WT, Movshon JA (1993) Responses of neurons in macaque MT to stochastic motion signals. Vis Neurosci 10:1157-1169.

Britten KH, Newsome WT, Shadlen MN, Celebrini S, Movshon JA (1996) A relationship between behavioral choice and the visual responses of neurons in macaque MT. Vis Neurosci 13:87-100.

Busse L, Katzner S, Tillmann C, Treue S (2008) Effects of attention on perceptual direction tuning curves in the human visual system. J Vis 8:2 1-13.

Carandini M, Ferster D (1997) A tonic hyperpolarization underlying contrast adaptation in cat visual cortex. Science 276:949-952.

Churchland MM, Yu BM, Cunningham JP, Sugrue LP, Cohen MR, Corrado GS, Newsome WT, Clark AM, Hosseini P, Scott BB, Bradley DC, Smith MA, Kohn A, Movshon JA, Armstrong KM, Moore T, Chang SW, Snyder LH, Lisberger SG, Priebe NJ, Finn IM, Ferster D, Ryu SI, Santhanam G, Sahani M, Shenoy KV (2010) Stimulus onset quenches neural variability: a widespread cortical phenomenon. Nat Neurosci 13:369-378.

Cohen MR, Maunsell JH (2009) Attention improves performance primarily by reducing interneuronal correlations. Nat Neurosci 12:1594-1600.

Cook EP, Maunsell JH (2002) Attentional modulation of behavioral performance and neuronal responses in middle temporal and ventral intraparietal areas of macaque monkey. J Neurosci 22:1994-2004.

Crook JM, Kisvárday ZF, Eysel UT (1998) Evidence for a contribution of lateral inhibition to orientation tuning and direction selectivity in cat visual cortex: reversible inactivation of functionally characterized sites combined with neuroanatomical tracing techniques. Eur J Neurosci 10: 2056-2075.

Deco G, Thiele A (2009) Attention: oscillations and neuropharmacology. Eur J Neurosci 30:347-354.

Deco G, Thiele A (2011) Cholinergic control of cortical network interactions enables feedback-mediated attentional modulation. Eur J Neurosci 34:146-157.

Disney AA, Domakonda KV, Aoki C (2006) Differential expression of muscarinic acetylcholine receptors across excitatory and inhibitory cells in visual cortical areas V1 and V2 of the macaque monkey. J Comp Neurol 499:49-63.

Disney AA, Aoki C, Hawken MJ (2007) Gain modulation by nicotine in macaque V1. Neuron 56:701-713.

Distler C, Hoffmann KP (2001) Cortical input to the nucleus of the optic tract and dorsal terminal nucleus (NOT-DTN) in macaques: a retrograde tracing study. Cereb Cortex 11:572-580.

Ghose GM (2009) Attentional modulation of visual responses by flexible input gain. J Neurophysiol 101:2089-2106.

Ghose GM, Maunsell JH (2002) Attentional modulation in visual cortex depends on task timing. Nature 419:616-620.

Gill TM, Sarter M, Givens B (2000) Sustained visual attention performanceassociated prefrontal neuronal activity: evidence for cholinergic modulation. J Neurosci 20:4745-4757.

Goard M, Dan Y (2009) Basal forebrain activation enhances cortical coding of natural scenes. Nat Neurosci 12:1444-1449.

Harris KD, Thiele A (2011) Cortical state and attention. Nat Rev Neurosci 12:509-523.

Herrero JL, Roberts MJ, Delicato LS, Gieselmann MA, Dayan P, Thiele A (2008) Acetylcholine contributes through muscarinic receptors to attentional modulation in V1. Nature 454:1110-1114.

Heuer HW, Britten KH (2002) Contrast dependence of response normalization in area MT of the rhesus macaque. J Neurophysiol 88:3398-3408.

Katzner S, Busse L, Carandini M (2011) GABA $A_{A}$ inhibition controls response gain in visual cortex. J Neurosci 31:5931-5941.

Khawaled R, Bruening-Wright A, Adelman JP, Maylie J (1999) Bicuculline block of small-conductance calcium-activated potassium channels. Pflugers Arch 438:314-321.

Kohn A, Movshon JA (2003) Neuronal adaptation to visual motion in area MT of the macaque. Neuron 39:681-691.

Lee J, Maunsell JH (2009) A normalization model of attentional modulation of single unit responses. PLoS One 4:e4651. 
Martínez-Trujillo J, Treue S (2002) Attentional modulation strength in cortical area MT depends on stimulus contrast. Neuron 35:365-370.

Martinez-Trujillo JC, Treue S (2004) Feature-based attention increases the selectivity of population responses in primate visual cortex. Curr Biol 14:744-751.

Maunsell JH, Van Essen DC (1983) Functional properties of neurons in middle temporal visual area of the macaque monkey. I. Selectivity for stimulus direction, speed and orientation. J Neurophysiol 49:1127-1147.

McAdams CJ, Maunsell JH (1999) Effects of attention on orientation tuning functions of single neurons in macaque cortical area V4. J Neurosci 19:431-441.

Metherate R, Tremblay N, Dykes RW (1988a) Transient and prolonged effects of acetylcholine on responsiveness of cat somatosensory cortical neurons. J Neurophysiol 59:1253-1276.

Metherate R, Tremblay N, Dykes RW (1988b) The effects of acetylcholine on response properties of cat somatosensory cortical neurons. J Neurophysiol 59:1231-1252.

Mitchell JF, Sundberg KA, Reynolds JH (2007) Differential attentiondependent response modulation across cell classes in macaque visual area V4. Neuron 55:131-141.

Mitchell JF, Sundberg KA, Reynolds JH (2009) Spatial attention decorrelates intrinsic activity fluctuations in macaque area V4. Neuron 63:879-888.

Movshon JA, Newsome WT (1996) Visual response properties of striate cortical neurons projecting to area MT in macaque monkeys. J Neurosci 16:7733-7741.

Movshon JA, Adelson EH, Gizzi M, Newsome WT (1985) The analysis of moving visual patterns. In: Study group on pattern recognition, Ed 1 (Chagas T, Gattass R, Gross CG, eds), pp 117-151. Vatican City, Italy: Pontifica Academia Scientiarum.

Murphy PC, Sillito AM (1991) Cholinergic enhancement of direction selectivity in the visual cortex of the cat. Neuroscience 40:13-20.

Murthy A, Humphrey AL (1999) Inhibitory contributions to spatiotemporal receptive-field structure and direction selectivity in simple cells of cat area 17. J Neurophysiol 81:1212-1224.

Niebergall R, Khayat PS, Treue S, Martinez-Trujillo JC (2011) Expansion of MT neurons excitatory receptive fields during covert attentive tracking. J Neurosci 31:15499-15510.

Parikh V, Sarter M (2008) Cholinergic mediation of attention: contributions of phasic and tonic increases in prefrontal cholinergic activity. Ann N Y Acad Sci 1129:225-235.

Parikh V, Kozak R, MartinezV, Sarter M (2007) Prefrontal acetylcholine release controls cue detection on multiple timescales. Neuron 56:141-154.

Reynolds JH, Heeger DJ (2009) The normalization model of attention. Neuron 61:168-185.

Reynolds JH, Chelazzi L, Desimone R (1999) Competitive mechanisms subserve attention in macaque areas V2 and V4. J Neurosci 19:1736-1753.

Reynolds JH, Pasternak T, Desimone R (2000) Attention increases sensitivity of V4 neurons. Neuron 26:703-714.

Roberts MJ, Zinke W, Guo K, Robertson R, McDonald JS, Thiele A (2005) Acetylcholine dynamically controls spatial integration in marmoset primary visual cortex. J Neurophysiol 93:2062-2072.

Roberts M, Delicato LS, Herrero J, Gieselmann MA, Thiele A (2007) Attention alters spatial integration in macaque $\mathrm{V} 1$ in an eccentricity-dependent manner. Nat Neurosci 10:1483-1491.

Roelfsema PR, Lamme VA, Spekreijse H (1998) Object-based attention in the primary visual cortex of the macaque monkey. Nature 395:376-381.

Roerig B, Kao JP (1999) Organization of intracortical circuits in relation to direction preference maps in ferret visual cortex. J Neurosci 19:RC44(1-5).

Sato H, Hata Y, Masui H, Tsumoto T (1987) A functional role of cholin- ergic innervation to neurons in the cat visual cortex. J Neurophysiol 58:765-780.

Sato H, Katsuyama N, Tamura H, Hata Y, Tsumoto T (1995) Mechanisms underlying direction selectivity of neurons in the primary visual cortex of the macaque. J Neurophysiol 74:1382-1394.

Sillito AM (1977) Inhibitory processes underlying the directional specificity of simple, complex and hypercomplex cells in the cat's visual cortex. J Physiol 271:699-720.

Sillito AM, Kemp JA (1983) Cholinergic modulation of the functional organization of the cat visual cortex. Brain Res 289:143-155.

Soma S, Shimegi S, Osaki H, Sato H (2012) Cholinergic modulation of response gain in the primary visual cortex of the macaque. J Neurophysiol 107:283-291.

Swindale NV (1998) Orientation tuning curves: empirical description and estimation of parameters. Biol Cybern 78:45-56.

Tassonyi E, Charpantier E, Muller D, Dumont L, Bertrand D (2002) The role of nicotinic acetylcholine receptors in the mechanisms of anesthesia. Brain Res Bull 57:133-150.

Thiele A, Hoffmann KP (2008) Neuronal firing rate, inter-neuron correlation and synchrony in area MT are correlated with directional choices during stimulus and reward expectation. Exp Brain Res 188:559-577.

Thiele A, Distler C, Hoffmann KP (1999) Decision-related activity in the macaque dorsal visual pathway. Eur J Neurosci 11:2044-2058.

Thiele A, Dobkins KR, Albright TD (2000) Neural correlates of contrast detection at threshold. Neuron 26:715-724.

Thiele A, Distler C, Korbmacher H, Hoffmann KP (2004) Contribution of inhibitory mechanisms to direction selectivity and response normalization in macaque middle temporal area. Proc Natl Acad Sci U S A 101:9810-9815.

Thiele A, Delicato LS, Roberts MJ, Gieselmann MA (2006) A novel electrode-pipette design for simultaneous recording of extracellular spikes and iontophoretic drug application in awake behaving monkeys. J Neurosci Methods 158:207-211.

Thiele A, Pooresmaeili A, Delicato LS, Herrero JL, Roelfsema PR (2009) Additive effects of attention and stimulus contrast in primary visual cortex. Cereb Cortex 19:2970-2981.

Treue S, Maunsell JH (1996) Attentional modulation of visual motion processing in cortical areas MT and MST. Nature 382:539-541.

Williford T, Maunsell JH (2006) Effects of spatial attention on contrast response functions in macaque area V4. J Neurophysiol 96:40-54.

Womelsdorf T, Anton-Erxleben K, Pieper F, Treue S (2006) Dynamic shifts of visual receptive fields in cortical area MT by spatial attention. Nat Neurosci 9:1156-1160.

Womelsdorf T, Anton-Erxleben K, Treue S (2008) Receptive field shift and shrinkage in macaque middle temporal area through attentional gain modulation. J Neurosci 28:8934-8944.

Yamakura T, Bertaccini E, Trudell JR, Harris RA (2001) Anesthetics and ion channels: molecular models and sites of action. Annu Rev Pharmacol Toxicol 41:23-51.

Zaksas D, Pasternak T (2006) Directional signals in the prefrontal cortex and in area MT during a working memory for visual motion task. J Neurosci 26:11726-11742.

Zinke W, Roberts MJ, Guo K, McDonald JS, Robertson R, Thiele A (2006) Cholinergic modulation of response properties and orientation tuning of neurons in primary visual cortex of anaesthetized marmoset monkeys. Eur J Neurosci 24:314-328.

Zohary E, Shadlen MN, Newsome WT (1994) Correlated neuronal discharge and its implication for psychophysical performance. Nature 370: $140-143$. 